\title{
Tunability of the spin reorientation transitions with pressure in $\mathrm{NdCO}_{5}$
}

Cite as: Appl. Phys. Lett. 116, 102408 (2020); https://doi.org/10.1063/1.5135640

Submitted: 07 November 2019 . Accepted: 02 March 2020 . Published Online: 13 March 2020

(D) Santosh Kumar, (iD Christopher E. Patrick, (iD) Rachel S. Edwards, (iD Geetha Balakrishnan, (D) Martin R. Lees, and (iD) Julie B. Staunton

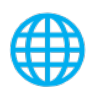

View Online

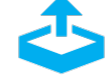

Export Citation

\section{ARTICLES YOU MAY BE INTERESTED IN}

Observing relaxation in device quality InGaN templates by TEM techniques

Applied Physics Letters 116, 102104 (2020); https://doi.org/10.1063/1.5139269

Thickness-dependent electron momentum relaxation times in iron films

Applied Physics Letters 116, 102406 (2020); https://doi.org/10.1063/1.5142479

Easy-cone magnetic structure in $\left(\mathrm{Cr}_{0.9} \mathrm{~B}_{0.1}\right) \mathrm{Te}$

Applied Physics Letters 116, 102404 (2020); https://doi.org/10.1063/5.0002118

\section{Challenge us.}

What are your needs for periodic signal detection?

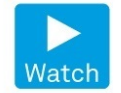

- Zurich

- Instruments

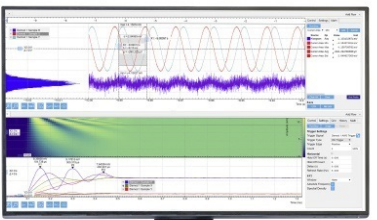

( 


\title{
Tunability of the spin reorientation transitions with pressure in $\mathrm{NdCO}_{5}$
}

\author{
Cite as: Appl. Phys. Lett. 116, 102408 (2020); doi: 10.1063/1.5135640 \\ Submitted: 7 November 2019 - Accepted: 2 March 2020 . \\ Published Online: 13 March 2020

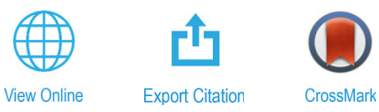

Santosh Kumar, ${ }^{\text {a) }}$ (D) Christopher E. Patrick, (D) Rachel S. Edwards, (D) Geetha Balakrishnan, (D) Martin R. Lees, ${ }^{\text {b) }}$ and Julie B. Staunton (D)

\author{
AFFILIATIONS \\ Department of Physics, University of Warwick, Coventry CV4 7AL, United Kingdom

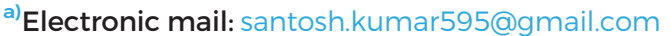 \\ b) Author to whom correspondence should be addressed: m.r.lees@warwick.ac.uk
}

\begin{abstract}
We present pressure-dependent magnetization measurements carried out in the domain of the spin reorientation transitions (SRTs) of a $\mathrm{NdCo}_{5}$ single crystal. The application of a hydrostatic pressure leads to a shift in the SRTs to higher temperatures. This shift is found to be very sensitive to pressure, with the SRT temperatures increasing at a rate of $\approx 17 \mathrm{~K} / \mathrm{GPa}$. To explain the experimental results, we have also performed first-principles calculations of the SRT temperatures for different applied strains, which corroborate the experimental findings. The calculations attribute the pressure dependence of the SRTs to a faster weakening of the Co contribution to the magnetocrystalline anisotropy with pressure compared to the $\mathrm{Nd}$ contribution.
\end{abstract}

Published under license by AIP Publishing. https://doi.org/10.1063/1.5135640

The class of intermetallic compounds with the formula $\mathrm{RCo}_{5}$ $(R=$ rare earth) are archetypal rare-earth transition-metal permanent magnets, displaying high Curie temperatures, large saturation magnetizations, and strong magnetic anisotropy, ${ }^{1}$ and have been the subject of intensive investigations both experimentally and theoretically for several decades. ${ }^{2-8} \mathrm{RCO}_{5}$ crystallizes in the hexagonal $\mathrm{P6} / \mathrm{mmm}$ structure with $\mathrm{RCo}_{2}$ planes interspersed with Co planes.' Magnetically, $R \mathrm{Co}_{5}$ is a ferrimagnet whose properties derive from two magnetic sublattices associated with rare earth and cobalt atoms. Competition between these sublattices can lead to unusual magnetic behavior, especially as a function of temperature. For example, in $\mathrm{GdCo}_{5}$, the $\mathrm{Gd}$ sublattice, whose moments are aligned antiparallel to the Co moments through an antiferromagnetic exchange interaction, disorders more quickly with temperature than the Co sublattice, and so the magnetization increases with temperature, reaching a peak around $800 \mathrm{~K} .^{7,10}$ This faster disordering of the $R$ sublattice is observed across the $R \mathrm{Co}_{5}$ series because of the weaker exchange interactions for $R$-Co than $\mathrm{Co}-\mathrm{Co} .{ }^{11}$ The antiferromagnetic exchange originates from a strong hybridization of the minority Co-3d and the $R-5 \mathrm{~d}$ bands. The majority Co-3d band, with lower energy, is unable to undergo this hybridization. ${ }^{12}$

The competition between the $R$ and Co sublattices also causes the magnetocrystalline anisotropy (MCA) to behave unusually. The MCA associated with the itinerant electrons forming the Co sublattice favors magnetization parallel to the crystallographic $c$-axis for all members of the $R \mathrm{Co}_{5}$ family. ${ }^{13}$ However, the MCA of the $R$ moments originates from the $R-4 f$ electrons interacting with the crystal field, and whether this interaction favors the $c$-axis or $a b$-plane magnetization depends on $R{ }^{14}$ Among the lighter rare earth elements, $R$ does not contribute to the MCA for $\mathrm{Y}$ and $\mathrm{La}$ and reinforces the $c$-axis anisotropy for Ce and Sm. Only in $\mathrm{PrCo}_{5}$ and $\mathrm{NdCo}_{5}$ does the $R$ contribution to the MCA favor $a b$ plane magnetization, competing with the uniaxial anisotropy of the Co moments. ${ }^{4}$ For $\mathrm{PrCo}_{5}$, the $\mathrm{Pr}$ contribution to the MCA is relatively weak, and so at cryogenic temperatures, the magnetization points $23^{\circ}$ from the $c$-axis and aligns along $c$ at temperatures above $105 \mathrm{~K}^{6}$ However, in $\mathrm{NdCo}_{5}$, the MCA associated with $\mathrm{Nd}$ is strong enough to overcome the Co anisotropy, and so the resultant magnetization lies in the $a b$ plane at low temperature. ${ }^{6,15}$

As the temperature of $\mathrm{NdCo}_{5}$ is raised, a faster disordering of the $R$ sublattice weakens the Nd planar MCA compared to the uniaxial Co contribution. At a critical temperature $T_{S R 1}$, the magnetization starts to rotate toward the $c$-axis (cone anisotropy). This rotation completes at $T_{\mathrm{SR} 2}$, when the magnetization points along the $c$-axis. The transitions from planar $\rightarrow$ cone and cone $\rightarrow c$-axis alignment are referred to as spin reorientation transitions (SRTs). ${ }^{16}$ The SRTs of $\mathrm{NdCo}_{5}$ have been the subject of a number of studies ${ }^{3,15-19}$ and are considered particularly interesting because $T_{\mathrm{SR} 1}$ and $T_{\mathrm{SR} 2}$ are not far from room temperature, at approximately 240 and $290 \mathrm{~K}$, respectively. This work includes our study of $\mathrm{NdCO}_{5}$ using torque magnetometry, investigating the underlying 
physics and location of the SRTs. ${ }^{15}$ Interest in $\mathrm{NdCo}_{5}$ was increased further by the discovery of a giant rotating magnetocaloric effect in $\mathrm{NdCO}_{5}$ with a maximum adiabatic temperature change observed at $280 \mathrm{~K}$, close to $T_{\mathrm{SR} 2}$, suggesting that this material could be used in magnetic refrigeration. ${ }^{20}$ This observation inspired attempts to bring the operating temperature of the magnetocaloric cycle closer to room temperature, by manipulating $T_{\mathrm{SR} 2}$. ${ }^{21,22}$

Any changes in the $R$ or Co MCA will affect the SRT temperatures. Crucially, however, since the SRT temperatures are determined by the balance between sublattices, it is the relative MCA enhancement that determines how the SRT temperatures change. Strengthening the $R$ contribution relative to Co will favor planar alignment over a wider temperature range increasing $T_{\mathrm{SR} 1}$ and $T_{\mathrm{SR} 2}$. Conversely, strengthening the Co uniaxial anisotropy relative to $R$ will decrease $T_{\mathrm{SR} 1}$ and $T_{\mathrm{SR} 2}$.

To date, attempts to change $T_{\mathrm{SR} 2}$ of $\mathrm{NdCo}_{5}$ have focused on introducing dopant atoms. ${ }^{21-25}$ For instance, substituting Co with B to form $\mathrm{NdCo}_{4} \mathrm{~B}$ increases the relative strength of the planar contribution to the extent that planar anisotropy dominates all the way to the Curie temperature so that no SRT is observed. ${ }^{25}$ Substitution with $\mathrm{Al}$ or $\mathrm{Si}$ to form $\mathrm{NdCo}_{4} \mathrm{Al}^{21}$ or $\mathrm{NdCo}_{4} \mathrm{Si}^{22}$ also shifts the balance in favor of planar anisotropy, but to a lesser extent than in $\mathrm{NdCo}_{4} \mathrm{~B}$. Accordingly, these compounds still exhibit SRTs and at elevated temperatures compared to $\mathrm{NdCO}_{5}$. However, the precise mechanism by which dopant atoms affect the SRTs is not easy to identify. Apart from modifying the electronic band structure, which will alter the Co anisotropy, the introduction of dopants will possibly change the crystal field at the $R$ site through both local interactions and also a global modification of the lattice parameters.

In this Letter, we demonstrate an alternative method to manipulate the SRTs by placing $\mathrm{NdCo}_{5}$ under hydrostatic pressure. Pressure is a "clean" variable that can bring about large changes in the structure and properties without altering the chemical composition. This makes high-pressure states amenable to the computational study. We show that applying pressure shifts the SRT temperatures upward, at a rate of $\sim 17 \mathrm{~K} / \mathrm{GPa}$. We complement the experimental measurements with finite temperature density-functional theory (DFT) calculations, which also find that the SRT temperatures increase with pressure, at a rate of $12 \mathrm{~K} / \mathrm{GPa}$. The calculations show how both the Nd and Co sublattice anisotropies are reduced as a result of applying pressure, but that relatively the decrease is stronger for Co, increasing the SRT temperatures.

The measurements were performed on a single crystal of $\mathrm{NdCo}_{5}$ grown using the optical floating zone technique. ${ }^{26}$ This compound has previously been prepared in different forms using other techniques. ${ }^{27,28}$ A crystal of $\mathrm{NdCo}_{5}$ with a volume of $\sim 0.05 \mathrm{~mm}^{3}$ was taken from the same batch used for our recent torque magnetometry study. ${ }^{15}$ The crystal was loaded into a cylindrical polytetrafluoroethylene sample holder filled with a pressure transmitting medium (Daphne oil) and placed in an easyLab Mcell 10 beryllium-copper piston clamp pressure cell. Care was taken to ensure that the sample would not move in a magnetic field. Hydrostatic pressure was applied at room temperature. The pressure in the cell was determined in situ from the superconducting transition temperature in a magnetic field of $1 \mathrm{mT}$ of a small piece of high purity (99.9999\%) tin placed alongside the sample. ${ }^{29}$ Measurements were carried out between 5 and $320 \mathrm{~K}$. The pressure set at room temperature varies by less than $10 \%$ over this temperature range. ${ }^{30}$ Magnetization measurements as a function of temperature were carried out using a Quantum Design Magnetic Property Measurement System magnetometer.
The strain- and temperature-dependent MCA of $\mathrm{NdCo}_{5}$ was calculated using the theory introduced in Ref. 31 . The $R-4 f$ electrons were treated within single-ion crystal field (CF) theory, where the CF coefficients were calculated from first principles using DFT. ${ }^{32}$ The Co moments were treated within the disordered local moment formulation of DFT (DFT-DLM), ${ }^{33}$ which takes into account the reduction of magnetic order as the temperature is increased. DFT-DLM calculations were also used to parameterize the exchange field entering the CF Hamiltonian, using the scheme introduced in Ref. 8. The same computational setup was used as in previous works, ${ }^{31,32}$ using the GPAW and Hutsepot codes. ${ }^{34,35}$ The easy direction of magnetization was calculated as a function of temperature and lattice parameters, where the experimental values of $a_{0}=5.0055 \AA$ and $c_{0}=3.9775 \AA$ as reported in Ref. 36 were used to define the zero-strain structure. Uniform compressive strains of $0.5,1.0$, and $2.0 \%$ were applied, where, for instance, a $1 \%$ compressive strain corresponds to lattice parameters $a=0.99 a_{0}, c=0.99 c_{0}$.

Figure 1(a) displays the temperature dependence of the magnetization, $M(T)$, for a $\mathrm{NdCO}_{5}$ crystal at various pressures. These data were collected while warming the sample at a rate of $1 \mathrm{~K} / \mathrm{min}$ in an applied field, $\mu_{0} H=0.05 \mathrm{~T}$. The SRT appears as a broad hump identifiable in all the $M(T)$ curves. The SRTs clearly shift to progressively higher temperatures as the applied pressure is increased up to $0.8 \mathrm{GPa}$, which is the maximum attainable with the current setup. In principle,
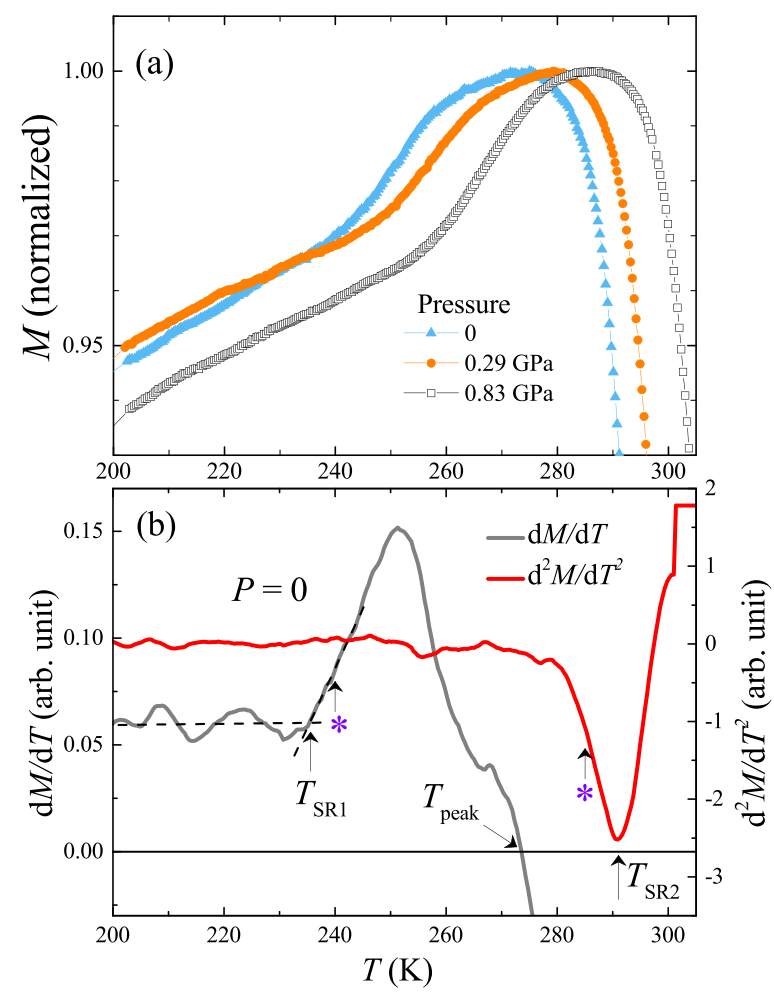

FIG. 1. (a) DC magnetization vs temperature, $M(T)$, for various applied pressures around the SRT region at a field, $\mu_{0} H=0.05 \mathrm{~T}$. (b) Temperature variation of first and second derivatives of $M(T)$ at ambient pressure. $T_{\mathrm{SR} 1}, T_{\text {peak }}$, and $T_{\mathrm{SR} 2}$ have been identified. The asterisks mark the corresponding $T_{\mathrm{SR} 1}$ (left) and $T_{\mathrm{SR} 2}$ (right) obtained at $\mu_{0} H=0.5 \mathrm{~T}$ from previous studies. 
torque magnetometry provides the clearest identification of temperatures of the SRTs, but unlike DC magnetization measurements, torque magnetometry cannot be performed inside a pressure cell. Instead, to obtain a precise location for $T_{\mathrm{SR} 1}$ and $T_{\mathrm{SR} 2}$, we show in Fig. 1(b) the first and second derivatives of the $M(T)$ curve recorded at ambient pressure. At $T_{\mathrm{SR} 1}$ (identified by the intersection of the dashed lines), there is a marked increase in $d M / d T$. This temperature nearly agrees with the $T_{\mathrm{SR} 1}$ (left asterisk) obtained from our torque measurements ${ }^{15}$ as well as from the reported magnetization data. ${ }^{16}$ The zero-crossing in $d M / d T$ corresponds to the temperature $\left(T_{\text {peak }}\right)$ where $M(T)$ is maximum, but there is no clear feature in $d M / d T$ to identify $T_{\mathrm{SR} 2}$. However, plotting the second derivative reveals a dip in $d^{2} M / d T^{2}$ that lies just above the $T_{\mathrm{SR} 2}$ (right asterisk) obtained from our torque study ${ }^{15}$ and the reported data. ${ }^{16}$ We use these two features-the rise in $d M / d T$ and the minimum in $d^{2} M / d T^{2}$ - as signatures of the SRTs. The two characteristic temperatures $\left(T_{\mathrm{SR} 1}\right.$ and $\left.T_{\mathrm{SR} 2}\right)$ identified at ambient pressure agree fairly well with our torque study ${ }^{15}$ that involved a different protocol of measurements.

DFT-DLM calculations corroborate the experimental measurements. Figure 2 shows the easy direction of magnetization, $\alpha$, calculated as a function of temperature for different compressive strains, where $\alpha$ is the polar angle with the $c$-axis. The SRT temperatures bound the region where $0^{\circ}<\alpha<90^{\circ}$. At zero strain, we calculate $T_{\mathrm{SR} 1}=213 \mathrm{~K}$ and $T_{\mathrm{SR} 2}=285 \mathrm{~K}$; the former is $23 \mathrm{~K}$ lower than the experimentally measured value at ambient pressure. Applying $0.5 \%$ compressive strain shifts the transition upwards by $35 \mathrm{~K}$. A further increase in the compressive strain increases the SRT temperatures, with $T_{\mathrm{SR} 2}$ reaching $438 \mathrm{~K}$ at $2 \%$ strain. We note that the calculated dependence is not linear, which could be a real effect or more likely numerical noise.

Figure 3(a) displays the experimentally measured variation with pressure of $T_{\mathrm{SR} 1}, T_{\text {peak }}$, and $T_{\mathrm{SR} 2}$. For reference, $T_{\mathrm{SR} 1}$ and $T_{\mathrm{SR} 2}$ identified from previous studies ${ }^{15,16}$ at ambient pressure are also shown in Fig. 3(a). Both $T_{\mathrm{SR} 1}$ and $T_{\mathrm{SR} 2}$ exhibit the same pressure dependence of $(17 \pm 3) \mathrm{K} / \mathrm{GPa}$. In Fig. $3(\mathrm{~b})$, we show the calculated $T_{\mathrm{SR} 2}$ values as a function of pressure. Rather than relying on DFT-calculated elastic properties, we use the experimentally measured volume vs pressure

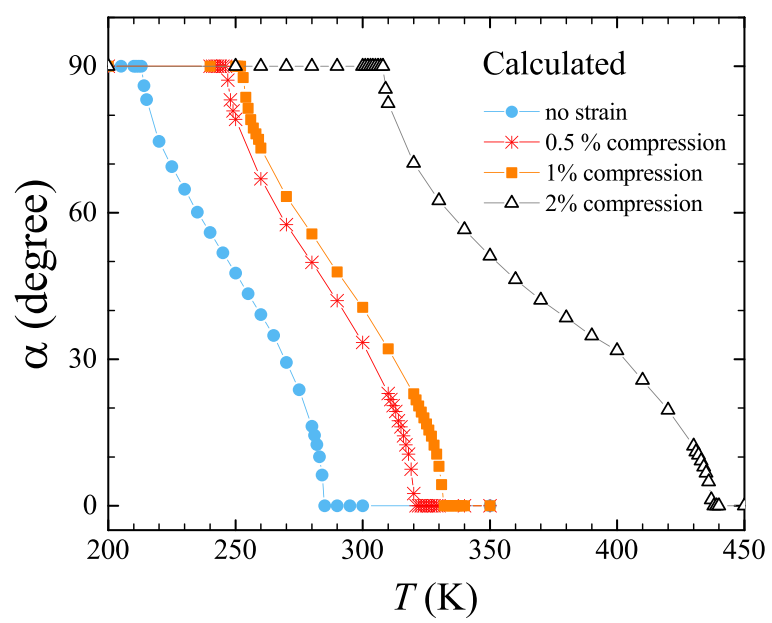

FIG. 2. Temperature variation of the easy angle of magnetization $\alpha$ calculated at different compressive strains.

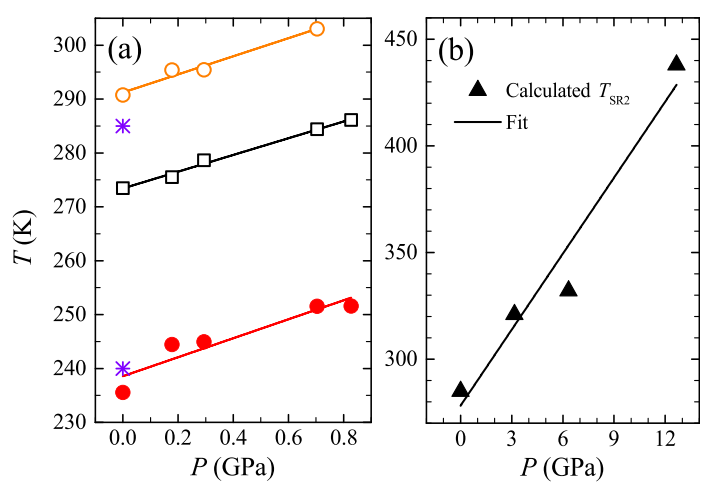

FIG. 3. (a) Pressure dependence of $T_{\mathrm{SR} 1}(\bigcirc), T_{\text {peak }}(\square)$, and $T_{\mathrm{SR} 2}(\bigcirc)$ obtained from experiments over the pressure range of $0 \leq P \leq 0.83 \mathrm{GPa}$. $T_{\mathrm{SR} 1}$ and $T_{\mathrm{SR} 2}$ from our torque magnetometry study ${ }^{15}$ at ambient pressure are marked by asterisks. $T_{\mathrm{SR} 2}$ could not be identified at $0.83 \mathrm{GPa}$ as it has shifted above the upper limit $(305 \mathrm{~K})$ of the measured temperature range. (b) Calculated $T_{\mathrm{SR} 2}$ vs the estimated pressure up to a maximum of $12 \mathrm{GPa}$.

curve of $\mathrm{YCo}_{5}$ reported in Ref. 37 to perform an approximate conversion of applied strain into a pressure of $6.3 \mathrm{GPa}$ per $1 \%$ strain. Clearly, the strains investigated computationally correspond to significantly larger pressures than those used experimentally, but smaller strains are more prone to numerical noise. A linear fit of the calculated $T_{\mathrm{SR} 2}$ values gives $12 \mathrm{~K} / \mathrm{GPa}$, in reasonable agreement with the experimental value of $(17 \pm 3) \mathrm{K} / \mathrm{GPa}$.

To explain the increase in the SRT temperatures with pressure, in Fig. 4, we plot the calculated strain dependence of the leading $\mathrm{CF}$

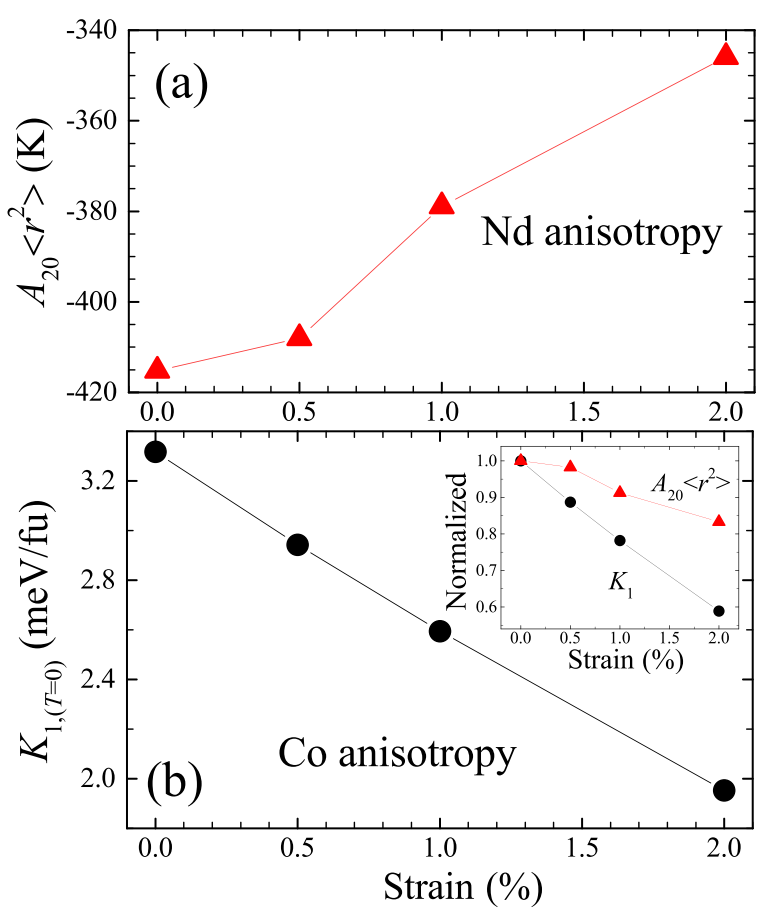

FIG. 4. Pressure dependence of (a) crystal field coefficients, $A_{20}\left\langle r^{2}\right\rangle$, calculated for the $\mathrm{Nd}$ sublattice and (b) the zero temperature anisotropy constants, $K_{1}$ of $\mathrm{Co}$. 
coefficient $A_{20}\left\langle r^{2}\right\rangle$ and the zero temperature anisotropy constant associated with the itinerant electrons, $K_{1} \cdot{ }^{31}$ These quantities provide the main contribution to the MCA associated with the Nd and Co sublattices, respectively. Both quantities decrease in magnitude as a compressive strain is applied, showing that the MCAs of both sublattices decrease with pressure. Indeed, we verified experimentally that $K_{1}$ decreases with pressure by performing magnetization measurements on $\mathrm{YCo}_{5}$, which, as $\mathrm{Y}$ is nonmagnetic, allows $K_{1}$ to be isolated. However, the aspect that is crucial to the SRTs is that the relative decrease with pressure is faster for $K_{1}$ than $A_{20}\left\langle r^{2}\right\rangle$. This behavior is shown in the inset of Fig. 4 where the quantities have been normalized to their zero pressure values. For instance, at $2 \%$ strain, $K_{1}$ has reduced by $42 \%$ compared to $A_{20}\left\langle r^{2}\right\rangle$, which has only reduced by $17 \%$. The increase in relative strength of the Nd MCA with pressure favors planar anisotropy over a wider temperature range, increasing $T_{\mathrm{SR} 1}$ and $T_{\mathrm{SR} 2}$.

In conclusion, both DC magnetization experiments and DFTDLM-based calculations have found that the SRT temperatures of $\mathrm{NdCo}_{5}$ increase with pressure. Furthermore, both methods find the rate of increase to be large, at $(17 \pm 3) \mathrm{K} / \mathrm{GPa}$ and $12 \mathrm{~K} / \mathrm{GPa}$, respectively. We note that an applied pressure of $1 \mathrm{GPa}$ is sufficient to raise the cone $\rightarrow c$-axis SRT-which coincides with the optimum temperature for a magnetocaloric cycle ${ }^{20}$ - to room temperature. The calculations explain the pressure dependence of the SRTs, with a faster decrease in the uniaxial magnetocrystalline anisotropy associated with the Co magnetic sublattice compared to the planar $\mathrm{Nd}$ contribution with increasing pressure. Our work demonstrates that the application of pressure may be used to modify the temperatures of the SRTs in both $\mathrm{NdCo}_{5}$ and other members of the $\mathrm{RCo}_{5}$ series in a controlled way. The observation that the magnetic phase is extremely sensitive to pressure should also motivate further study of $\mathrm{NdCo}_{5}$ as a potential barocaloric material. ${ }^{38}$

See the supplementary material for the details of magnetization measurements performed under pressure on a single crystal of $\mathrm{YCo}_{5}$, which show that $K_{1}$ in $\mathrm{YCo}_{5}$ decreases with pressure.

This work is a part of the PRETAMAG project funded by the Engineering and Physical Sciences Research Council, Grant Nos. $\mathrm{EP} / \mathrm{M} 028941 / 1$ and EP/M028771/1. M. Ciomaga Hatnean is acknowledged for assistance with single crystal growth. We thank G. A. Marchant for useful discussions.

\section{REFERENCES}

${ }^{1}$ K. Strnat, G. Hoffer, J. Olson, W. Ostertag, and J. J. Becker, J. Appl. Phys. 38, 1001 (1967).

${ }^{2}$ K. J. H. Buschow and M. Brouha, J. Appl. Phys. 47, 1653 (1976).

${ }^{3}$ H. P. Klein, A. Menth, and R. S. Perkins, Physica 80B, 153 (1975).

${ }^{4}$ A. Ermolenko, IEEE Trans. Magn. 12, 992 (1976).

${ }^{5}$ H. Yoshie, K. Ogino, H. Nagai, A. Tsujimura, and Y. Nakamura, J. Magn. Magn. Mater. 70, 303 (1987).

${ }^{6}$ E. Tatsumoto, T. Okamoto, H. Fujii, and C. Inoue, J. Phys. Colloq. 32, C1 550 (1971).

${ }^{7}$ C. E. Patrick, S. Kumar, G. Balakrishnan, R. S. Edwards, M. R. Lees, E. Mendive-Tapia, L. Petit, and J. B. Staunton, Phys. Rev. Mater. 1, 024411 (2017).

${ }^{8}$ C. E. Patrick, S. Kumar, G. Balakrishnan, R. S. Edwards, M. R. Lees, L. Petit, and J. B. Staunton, Phys. Rev. Lett. 120, 097202 (2018).

${ }^{9}$ J. H. Wernick and S. Geller, Acta Crystallogr. 12, 662 (1959).
${ }^{10}$ A. S. Yermolenko, Fiz. Met. Metalloved. 50, 741 (1980).

${ }^{11}$ C. E. Patrick and J. B. Staunton, Phys. Rev. B 97, 224415 (2018).

${ }^{12}$ M. S. S. Brooks, O. Eriksson, and B. Johansson, J. Phys.: Condens. Matter 1, 5861 (1989).

${ }^{13}$ Z. Tie-song, J. Han-min, G. Guang-hua, H. Xiu-feng, and C. Hong, Phys. Rev. B 43, 8593 (1991).

${ }^{14}$ M. D. Kuz'min and A. M. Tishin, "Theory of crystal field effects in $3 d-4 f$ intermetallic compounds, in handbook of magnetic materials," in Handbook of Magnetic Materials, edited by K. H. J. Buschow (Elsevier B.V., 2008), Vol. 17, Chap. 3, p. 149.

${ }^{15}$ S. Kumar, C. E. Patrick, R. S. Edwards, G. Balakrishnan, M. R. Lees, and J. B. Staunton, "Torque magnetometry study of the pin reorientation transition and temperature-dependent magnetocrystalline anisotropy in NdCo5," J. Phys.: Condens. Matter (to be published).

${ }^{16}$ M. M. Ohkoshi, H. Kobayshi, T. Katayama, M. Hirano, T. Katayama, and T. Tsushima, AIP Conf. Proc. 29, 616 (1976).

${ }^{17}$ J. B. Sousa, A. Moreira, J. M. Del Moral, P. Algarabel, and R. Ibarra, J. Phys.: Condens. Matter 2, 3897 (1990).

${ }^{18}$ M. Seifert, L. Schultz, R. Schäfer, V. Neu, S. Hankemeier, S. Rössler, R. Frömter, and H. P. Oepen, New J. Phys. 15, 013019 (2013).

${ }^{19}$ H. Bartholin, B. Van Laar, R. Lemaire, and J. Schweizer, J. Phys. Chem. Solids 27, 1287 (1966)

${ }^{20}$ S. A. Nikitin, K. P. Skokov, Y. S. Koshkid'ko, Y. G. Pastushenkov, and T. I. Ivanova, Phys. Rev. Lett. 105, 137205 (2010).

${ }^{21}$ Y. Hu, Q. B. Hu, C. C. Wang, Q. Q. Cao, W. L. Gao, D. H. Wang, and Y. W. Du, Solid State Commun. 250, 45 (2017).

${ }^{22}$ K. Wang, M. Zhang, J. Liu, H. Luo, and J. Sun, J. Appl. Phys. 125, 243901 (2019).

${ }^{23}$ M. Sagawa, W. Yamagishi, and Z. Henmi, J. Appl. Phys. 52, 2520 (1981).

${ }^{24}$ S. C. Ma, D. H. Wang, C. L. Zhang, H. C. Xuan, S. D. Li, Z. G. Huang, and Y. W. Du, J. Alloys Compd. 499, 7 (2010).

${ }^{25}$ H. Ido, W. E. Wallace, T. Suzuki, S. F. Cheng, V. K. Sinha, and S. G. Sankar, T. Appl. Phys. 67, 4635 (1990).

${ }^{26}$ R. P. Singh, M. Smidman, M. R. Lees, D. McK. Paul, and G. Balakrishnan, J. Cryst. Growth 361, 129 (2012).

${ }^{27}$ F. Valdés-Bango, F. J. García Alonso, G. Rodríguez-Rodríguez, L. Morán Fernandez, A. Anillo, L. Ruiz-Valdepeñas, E. Navarro, J. L. Vicent, M. Vélez, J. I. Martín, and J. M. Alameda, J. Appl. Phys. 112, 083914 (2012).

${ }^{28}$ A. Hierro-Rodriguez, J. M. Teixeira, M. Vélez, L. M. Alvarez-Prado, J. I. Martín, and J. M. Alameda, Appl. Phys. Lett. 105, 102412 (2014).

${ }^{29}$ L. D. Jennings and C. A. Swenson, Phys. Rev. B 112, 31 (1958).

${ }^{30}$ ML04 03e-Mcell 10 Technical Note, Almax-easyLab (2013).

${ }^{31}$ C. E. Patrick and J. B. Staunton, Phys. Rev. Mater. 3, 101401 (2019).

${ }^{32}$ C. E. Patrick and J. B. Staunton, J. Phys.: Condens. Matter 31, 305901 (2019).

${ }^{33}$ B. L. Györffy, A. J. Pindor, J. Staunton, G. M. Stocks, and H. Winter, J. Phys. F 15, 1337 (1985).

${ }^{34}$ J. Enkovaara, C. Rostgaard, J. J. Mortensen, J. Chen, M. Dułak, L. Ferrighi, J. Gavnholt, C. Glinsvad, V. Haikola, H. A. Hansen, H. H. Kristoffersen, M. Kuisma, A. H. Larsen, L. Lehtovaara, M. Ljungberg, O. Lopez-Acevedo, P. G. Moses, J. Ojanen, T. Olsen, V. Petzold, N. A. Romero, J. Stausholm-Møller, M. Strange, G. A. Tritsaris, M. Vanin, M. Walter, B. Hammer, H. Häkkinen, G. K. H. Madsen, R. M. Nieminen, J. K. Nørskov, M. Puska, T. T. Rantala, J. Schiøtz, K. S. Thygesen, and K. W. Jacobsen, J. Phys.: Condens. Matter 22, 253202 (2010).

${ }^{35}$ M. Däne, M. Lüders, A. Ernst, D. Ködderitzsch, W. M. Temmerman, Z. Szotek, and W. Hergert, J. Phys.: Condens. Matter 21, 045604 (2009).

${ }^{36}$ A. V. Andreev, "Thermal expansion anomalies and spontaneous magnetostriction in rare-earth intermetallics with cobalt and iron," in Handbook of Magnetic Materials, edited by K. H. J. Buschow (Elsevier, North-Holland, New York, 1995), Vol. 8, Chap. 2, p. 59.

${ }^{37}$ D. Koudela, U. Schwarz, H. Rosner, U. Burkhardt, A. Handstein, M. Hanfland, M. D. Kuz'min, I. Opahle, K. Koepernik, K.-H. Müller, and M. Richter, Phys. Rev. B 77, 024411 (2008).

${ }^{38}$ M. V. Gorev, E. V. Bogdanov, and I. N. Flerov, J. Phys. D: Appl. Phys. 50, 384002 (2017). 